\title{
From Scanning Electron Nanodiffraction to 4D-STEM: How and Why Coherence Matters
}

\author{
Jian-Min Zuo, Renliang Yuan and Haw-Wen Hsiao
}

University of Illinois at Urbana-Champaign, Urbana, Illinois, United States

In the recent history of electron microscopy, electron detectors have played critical role, from the development of charge coupled device (CCD) camera1, which replaced the film as the method of choice for image recording, to direct detection camera that revolutionized cryogenic electron microscopy 2. Similarly, the development of fast electron pixel array detectors has generated considerable excitement in scanning transmission electron microscopy (STEM)3, leading to an assortment of imaging techniques that are enabled by recording 4D data, namely, the electron wave intensity in the momentum space (kx, ky) for each probe position in the real space ( $\mathrm{x}, \mathrm{y}$ ) (thus 4D-STEM)4. The electron wave intensity is analyzed and related to sample structure, or electric and magnetic fields that all impact on the electron wave function. As the speed of STEM pixel array detectors improves dramatically, 4D-STEM increasingly becomes the method of choice for STEM imaging. New breakthroughs come with single electron counting, fast readout and large dynamic range. While the detector size (number of pixels) is still relatively small compared to electron cameras designed for electron diffraction, but the size is significantly larger than the segmented STEM detectors, used for Center of Mass (COM) imaging, for example5. What is and is not measured matter greatly for 4D-STEM. While the detector requirements are relatively modest for applications such as phase and orientation mapping, where the measurement of a few diffraction spots in approximate intensity scales often suffices, applications of high precision requires resolution in momentum space or the measurement of elastic scattering intensity with energy filtering. Examples include strain mapping, where large format detectors are required, and dynamical inversion, where filtering of inelastic background is often necessary except very thin samples. Perhaps, the community as whole has paid less attention to the role of coherence. In this aspect, it is worthwhile to revisit the work of late Professor John Cowley, who pioneered the technique of electron nanodiffraction (END), using a focused probe realized in a field emission gun (FEG) STEM as a means for obtaining information on structural detail, in the range between that of STEM image resolution and the limits of information possible from elastic scattering6. The probe convergence angle and electron lateral coherence determine how and where electron interferes, as expressed in following equation in the limit of kinematical scattering and perfect coherence (1) where is the electron probe wave function and is the crystal potential. The extent of crystal potential seen by the electron probe is determined by electron lateral coherence length (Lc), which is determined by the virtual electron source size [ref], and the extent of electron wave function (Lp), which is determined by the probe convergence angle and lens aberrations. With infinite coherence length ( $\mathrm{Lc}>>\mathrm{Lp}$ ), the convolution in eq. 1 places the reciprocal electron probe wave function on each reciprocal lattice point, their overlap is the basis for atomic resolution imaging in STEM (Fig. 1). The interference occurs in the overlapping region between Bragg diffraction disks for Bragg Interferometry. With finite coherence length (Lc Lp) or for an imperfect crystal, the reciprocal lattice point broadens, the resulted overlap of electron probe wave function causes interference at small scattering angles that can only be resolved at a large camera length. Such interference provides the sensitivity to subtle changes in crystal structure such as small lattice strain, which is fundamentally different from Bragg interferometry of STEM imaging. While the reciprocal lattice point broadening is typically observed as diffuse scattering in selected area electron diffraction, the development of scanning electron nanodiffraction (SEND) enables the mapping of local structural information. As such, SEND performed with a coherent probe and a high-resolution detector extends the measurement of local small angle scattering across the length scale 
of hundreds of nm, which makes it a truly exceptional diffraction technique for the characterization of imperfect crystals.

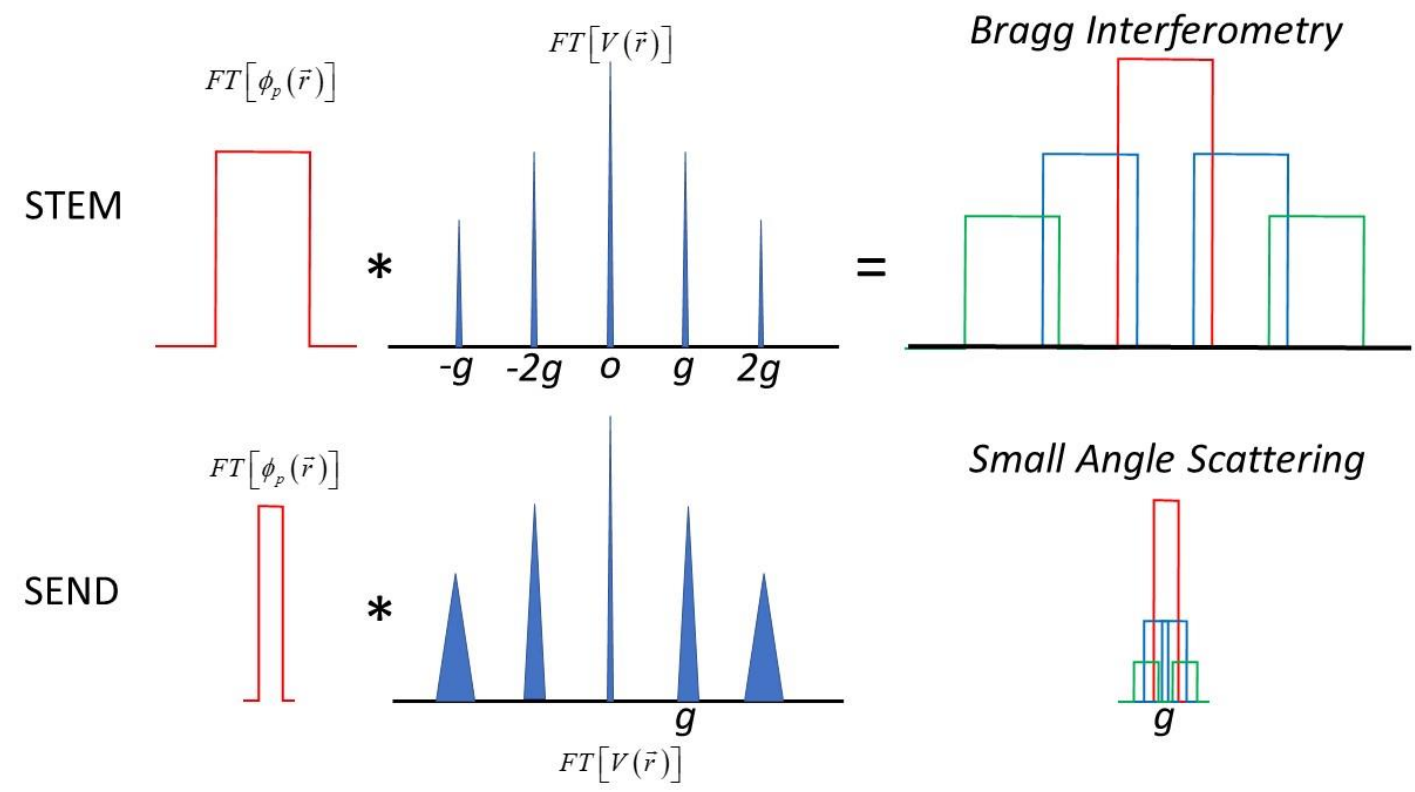

Figure 1. Interference in STEM and SEND.

References

1 J. C. H. Spence and J. M. Zuo, Review of Scientific Instruments 59 (9), 2102 (1988).

2 A. R. Faruqi, R. Henderson, M. Pryddetch, P. Allport, and A. Evans, Nuclear Instruments \& Methods in Physics Research Section A 546 (1-2), 170 (2005).

$3 \quad$ Mark W. Tate et al., Microscopy and Microanalysis 22 (1), 237 (2016); $\quad$ Matus Krajnak, Damien McGrouther, Dzmitry Maneuski, Val O. Shea, and Stephen McVitie, Ultramicroscopy 165, 42 (2016).

$4 \quad$ Colin Ophus, Microscopy and Microanalysis 25 (3), 563 (2019).

$5 \quad$ Naoya Shibata, Scott D. Findlay, Yuji Kohno, Hidetaka Sawada, Yukihito Kondo, and Yuichi Ikuhara, Nature Physics 8 (8), 611 (2012).

6 J. M. Cowley, Microscopy Research and Technique 46 (2), 75 (1999). 